\title{
Clinical and Hematological Predictors of High-Grade Immune-Related Adverse Events Associated With Immune Checkpoint Inhibitors
}

\author{
Ashish Manne $^{\mathrm{a}, \mathrm{h}} \mathbb{D}$, Madhuri S. Mulekar ${ }^{\mathrm{b}}$, Daisy E. Escobar ${ }^{\mathrm{c}}$, Alhareth Alsayed ${ }^{\mathrm{d}}$, \\ Gaurav Sharmac, Pranitha Prodduturvare, Moh'd Khushman ${ }^{\mathrm{d}}$, John Harrison Howardf, \\ Robert Gilbert ${ }^{\mathrm{g}}$, Omar Alkharabsheh ${ }^{\mathrm{d}}$
}

\begin{abstract}
Background: Life-threatening immune-related adverse events (irAEs) that require hospital admission are not uncommon in patients treated with immune checkpoint inhibitors (ICIs). The clinical and hematological parameters are attractive biomarkers as potential predictors of irAE.

Methods: This is a retrospective study of patients with melanoma and lung cancer treated with ICIs between 2015 and 2019 at the University of South Alabama Mitchell Cancer Institute. Fisher's exact test, Pearson Chi-squared test, log-rank test, and Cox proportional hazard model were used to evaluate clinical and hematological parameters as possible predictors of irAE.
\end{abstract}

Results: The cohort consisted of 160 patients treated with at least two doses of ICI, of which 54 (33.8\%) patients had melanoma and 106 $(66.3 \%)$ had lung cancer. Incidence of irAE did not have any bearing on the overall survival (OS) or progression-free survival (PFS) of the cohort. The clinical factors associated with irAE were dual-agent therapy (ipilimumab/nivolumab combination) and high disease burden ( $\geq 2$ metastatic sites). The irAE-group had a lower mean platelet-

Manuscript submitted April 21, 2021, accepted May 4, 2021

Published online May 25, 2021

aDivision of Medical Oncology, Department of Internal Medicine, James Comprehensive Cancer Center, Ohio State University, Columbus, OH 43210, USA

bepartment of Mathematics and Statistics, The University of South Alabama, Mobile, AL, USA

'Department of Medicine, The University of South Alabama, Mobile, AL, USA

${ }^{\mathrm{d}}$ Division of Medical Oncology, The University of South Alabama, Mitchell Cancer Institute, Mobile, AL, USA

eDivision of Medical Oncology, Mayo Clinic, Rochester, MN, USA

fDepartment of Surgery, The University of South Alabama, Mobile, AL, USA gDivision of Radiation Oncology, The University of South Alabama, Mobile, AL, USA

${ }^{\mathrm{h} C}$ Corresponding Author: Ashish Manne, Division of Medical Oncology, Department of Internal Medicine, James Comprehensive Cancer Center, Ohio State University, B407A Starling Loving Hall, 320 West 10th Avenue, Columbus, OH 43210, USA. Email: manne.ashish@gmail.com

doi: https://doi.org/10.14740/jocmr4511 to-lymphocyte ration (PLR, 200 vs. 257, P=0.04). Although not statistically significant at the level of 0.05 , other factors such as type of cancer (lung cancer $>$ melanoma $(P=0.06)$ ), stage at treatment (stage IV $>$ stage II and III disease $(\mathrm{P}=0.06))$, and higher absolute lymphocyte counts $(\mathrm{P}=0.07)$ showed a considerable association with irAE and warrants further review with different patient data.

Conclusions: Irrespective of ICI used to treat lung cancer and melanoma, patients with high disease burden and dual-agent ICI therapy were more prone to irAE. The only hematological parameter that may predict the incidence of irAE is low baseline PLR.

Keywords: Checkpoint inhibitors; Immune-related adverse events; Lymphocytes; Predictors of toxicity; Lung cancer; Melanoma; Hematological risk factors

\section{Introduction}

The interest in the factors to predict response and incidence of immune-related adverse events (irAEs) has grown in recent years, given the exponential growth of immune checkpoint inhibitors (ICIs) used in clinical practice. Tumor mutational burden, level of programmed death-ligand 1 (PD-L1) expression, microsatellite instability (MSI), baseline size of the primary tumor, and type of the cancer are some of the clinical factors that have limited utility in predicting the response to ICIs; however, there are no well-established factors that can predict the irAE [1-5]. The value of pretreatment complete blood counts, including absolute lymphocyte count (ALC), neutrophil-to-lymphocyte ratio (NLR), or platelet-to-lymphocyte ratio (PLR), for this purpose is equivocal. A few retrospective studies have demonstrated that lymphopenia is associated with early ICI treatment failure, whereas others attributed higher $\operatorname{ALC}\left(>2,000 / \mathrm{mm}^{3}\right)$ with the increased risk of irAE. The link between irAE and the efficacy of ICIs is also ambiguous, with no clear correlation to predict better or worse outcomes [6-8].

Some biomarkers, such as interleukin 17 (IL-17), eosinophil count, combined toxicity scores based on gene expression, autoantibodies, and gut microbiota, have shown correlative results with the incidence of irAE [9-14]. However, none of 
them are used in current clinical practice. Combining ICI with chemotherapy or targeted therapy might confound irAE predictive markers $[15,16]$. Glucocorticoids and holding ICI are the mainstay of management of irAE, whereas other immunosuppressive agents, like infliximab or mycophenolate, are used after glucocorticoid failure [17].

In patients who develop irAE while having an appropriate clinical response to ICI, restarting ICI post irAE management requires careful consideration of the risks and benefits of ICI. Factors that challenge restarting ICI after irAE are underlying comorbidities, such as the presence of autoimmune diseases, the severity of irAE, involved organ, glucocorticoid failure, decline in performance status secondary to prolonged irAE management, and high-dose glucocorticoids [18-21]. In clinical practice, patients who achieve complete recovery of irAE with no recurrence while on glucocorticoid taper are considered candidates for ICI re-initiation [22].

In this study, lung cancer (adenocarcinoma, squamous, and small cell lung carcinoma) and melanoma treated with ICIs were investigated to identify tumor charectestics clinical and hematological factors that predict the incidence of lifethreatening irAE. The goal was to optimize patient monitoring and possibly recognize the critical time to hold ICIs before progression to severe irAE that requires hospitalization.

\section{Materials and Methods}

After receiving appropriate institutional review board approvals, electronic health records of the University of South Alabama Mitchell Cancer Institute (August 2015 through February 2020) were searched using the diagnosis code from the International Statistical Classification of Diseases (ICD) 10 th version (i.e., ICD10 code) and the immunotherapy drug names. All the present study procedures were conducted in compliance with the Helsinki Declaration for research on human beings. The local research ethics committee approved the study. Lung cancer and melanoma were selected for this study, as they are the top two solid tumors treated with ICIs in the cancer center. Patients included in this study had only one (current) primary cancer (either lung or melanoma), received at least two doses of ICI, and had necessary baseline laboratory data, including complete blood count with white blood cell (WBC) differential before the first dose of treatment. Patients with a history of different cancer within the last 5 years or those who received more than one type of immunotherapy drug (except dual-agent ICI protocols) or had insufficient baseline data were excluded.

Data extracted retrospectively included: 1) demographics, such as age, race, and sex; 2) clinical data of the primary malignancy, stage, number of metastatic sites, and prior systemic therapy; 3) complete blood counts, including total WBC, absolute neutrophilic count (ANC), ALC, platelet count, and hemoglobin; and 4) the type of ICI (single-agent vs. dual-agent).

In this study, irAE is synonymous with life-threatening high-grade irAE that required hospitalization. The irAE grade was determined according to the National Cancer Institute Common Terminology Criteria for Adverse Events (CTCAE) v.5.0 [23]. In patients who developed irAE, hematological parameters at the time of the event and the last follow-up were determined. PLR was calculated as a ratio of the platelet count to ALC, and the NLR was calculated as a ratio of ANC to ALC. Non-metastatic cancers and metastatic cancers with only one organ metastasis were considered a low-burden disease, whereas metastatic cancers with more than one $(\geq 2)$ metastatic site (organ) were considered a high-burden disease. In this study, dual-agent ICI therapy refers to the combination of ipilimumab and nivolumab.

In order to compare the clinical factors that influence the incidence of irAEs, the entire cohort was divided into two groups: 1) the irAE group, which included subjects who had at least one episode of high-grade irAE; and 2) non-irAE group, which included subjects without a history of irAE. For analysis, primary cancer was classified as lung versus melanoma, age at diagnosis as $<65$ versus $\geq 65$ years, stage as metastatic versus non-metastatic, and disease burden as low versus high. Also, WBC threshold of $10 / \mathrm{mm}^{3}$ (i.e., 10 vs. $>10$ ), ALC thresholds of $600 / \mathrm{mm}^{3}, 1,000 / \mathrm{mm}^{3}$, and $2,000 / \mathrm{mm}^{3}$, and PLR threshold (i.e., $\leq 150$ and $>150$ ) were used in the analysis, based on previously published literature [24, 25].

Data were analyzed using statistical software JMP Pro 14.2 (SAS Institute Inc., Cary, NC). Pearson's Chi-squared test or Fisher's test was used to examine the association between nominal variables. Means of continuous variables, such as total WBC count, ALC, ANC, NLR, and PLR, were compared by Student's $t$-test or analysis of variance (ANOVA). A matched pairs $t$-test was used to study the mean change in hematological parameters between the baseline, at the time of irAE incidence, and last available values. Association between continuous variables was studied using Pearson's correlation coefficient. Multivariate logistic regression was used to study association between the occurrence/non-occurrence of adverse events and clinical and hematological factors. For the entire cohort, the median overall survival and a 95\% confidence interval (CI) were estimated using the Kaplan-Meier method. The log-rank test was used to compare median survival rates for different groups. The difference in the distributions of PFS and OS were assessed for various risk factors. Results with $\mathrm{P}$ value $<0.05$ were considered statistically significant.

\section{Results}

The search of the electronic medical records using the ICD10 code and drug name yielded 188 patient records. After applying the inclusion and exclusion criteria, a total of 160 patients (106 with lung cancer, and 54 with melanoma) were included in the study. The baseline characteristics of the cohort are summarized in Table 1. The median age of the cohort was 64 years (range: 17 - 93 years), and most patients were Caucasians $(76.3 \%)$. ICI was administered to $48(30.0 \%)$ patients with recurrence, whereas $62(38.8 \%)$ patients had received a prior systemic treatment (mostly in lung cancer using carboplatin, paclitaxel, pemetrexed, nab-paclitaxel, and etoposide). In $38(23.8 \%)$ patients, ICI was combined with chemotherapy. Disease progression (PD) occurred in 54 (33.8\%), and irAE 
Table 1. Baseline Characteristics of the Cohort

\begin{tabular}{|c|c|}
\hline Patient characteristics $(n=160)$ & $\begin{array}{l}\mathrm{N}(\%) \text { or median (mini- } \\
\text { mum - maximum) }\end{array}$ \\
\hline Age at diagnosis (years) & $64(17-93)$ \\
\hline \multicolumn{2}{|l|}{ Gender } \\
\hline Male & $79(49.4 \%)$ \\
\hline Female & $81(50.6 \%)$ \\
\hline \multicolumn{2}{|l|}{ Race } \\
\hline African American & $38(23.8 \%)$ \\
\hline Caucasian & $122(76.2 \%)$ \\
\hline \multicolumn{2}{|l|}{ Diagnosis } \\
\hline Lung cancer & $106(66.3 \%)$ \\
\hline Melanoma & $54(63.7)$ \\
\hline \multicolumn{2}{|l|}{ Stage } \\
\hline Stage II or III & $57(35.7 \%)$ \\
\hline Stage IV & $103(64.4 \%)$ \\
\hline \multicolumn{2}{|l|}{ Visceral metastatic disease } \\
\hline None & $57(36 \%)$ \\
\hline Single metastatic site & $23(14 \%)$ \\
\hline$\geq 2$ metastatic sites & $80(50 \%)$ \\
\hline \multicolumn{2}{|l|}{ Disease burden } \\
\hline Low & $80(50 \%)$ \\
\hline High & $80(50 \%)$ \\
\hline \multicolumn{2}{|l|}{ Status at last check } \\
\hline Alive & $130(81.3 \%)$ \\
\hline Diseased & $30(18.8 \%)$ \\
\hline \multicolumn{2}{|l|}{ LN only } \\
\hline No & $134(83.7 \%)$ \\
\hline Yes & $26(16.3 \%)$ \\
\hline \multicolumn{2}{|l|}{ Previous systemic treatment } \\
\hline Yes & $62(38.7 \%)$ \\
\hline No & $98(61.3 \%)$ \\
\hline \multicolumn{2}{|l|}{ Combination with chemotherapy } \\
\hline Yes & $38(23.7 \%)$ \\
\hline No & $122(76.3 \%)$ \\
\hline \multicolumn{2}{|l|}{ ICI and the line of treatment } \\
\hline First-line & $48(30.0 \%)$ \\
\hline Second-line & $112(70.0 \%)$ \\
\hline \multicolumn{2}{|l|}{ Treatment agents } \\
\hline Ipilimumab only & $5(3.14 \%)$ \\
\hline Nivolumab only & $47(29.56 \%)$ \\
\hline Ipilimumab + nivolumabo & $31(19.50 \%)$ \\
\hline Pembrolizumab & $55(34.59 \%)$ \\
\hline Durvalumab & $14(8.81 \%)$ \\
\hline Atezolizumab & $7(4.40 \%)$ \\
\hline
\end{tabular}

Categorical variables are summarized using the number (percentage), and numerical variables are summarized using median (minimum maximum). LN: lymph node; ICI: immune checkpoint inhibitor. was reported in $46(28.8 \%)$ patients.

In our cohort, the censored median PFS and OS values for different patient characteristics are listed here (Supplemental Material 1, www.jocmr.org). Significant difference was observed in the PFS distributions of patients with lung cancer versus melanoma $(\mathrm{P}=0.02)$, those with ICIs in firstline versus second-line therapy $(\mathrm{P}=0.0002)$, with or without combination chemotherapy $(\mathrm{P}=0.002)$, low versus high level of disease burden $(\mathrm{P}=0.01)$, and $\mathrm{WBC} \leq 10\left(\times 10^{9} / \mathrm{L}\right)$ versus $>10\left(\times 10^{9} / \mathrm{L}, \mathrm{P}=0.02\right)$. On the other hand, no significant differences in the OS distributions by patient characteristics were observed. The median PFS was observed to be higher for patients treated with ICIs in second line, alone (without chemotherapy), and $\mathrm{WBC} \leq 10 \times 10^{9} / \mathrm{L}$. The distribution of PFS based on type of cancer, line of treatment (primary $=$ first line and recurrence $\mathrm{Rx}=$ second line), WBC count, combination of chemotherapy with ICI, and disease burden are illustrated here (Supplement Material 2, www.jocmr.org).

\section{$\operatorname{ir} \mathbf{A E}$}

Out of 160 patients in the cohort, $46(28.8 \%)$ had at least one high-grade irAE requiring admission. This irAE-group of 46 patients was fairly evenly divided by sex ( 21 females and 25 males), diagnosis (25 lung cancer vs. 21 melanoma), and age group (21 younger than 65 years and 25 of age $65+$ ), but consisted of 39 (84.8\%) Caucasian patients, 35 (76.1\%) stage IV patients, 29 (63.0\%) high-burden patients, and 31 (67.4\%) patients treated with ICIs in the first line. All patients received at least one dose of methylprednisolone $1 \mathrm{mg} / \mathrm{kg}$ or equivalent on the day of admission, while infliximab had to be used in six patients. None of the patients had infliximab-refractory irAE. Baseline characteristics between the irAE and non-irAE groups are illustrated in Table 2.

\section{Comparing the irAE and non-irAE groups}

The incidence of irAE did not influence both the OS $(\mathrm{P}=0.18)$ and PFS $(\mathrm{P}=0.76)$ in the entire cohort (Supplement Material 1, www.jocmr.org). The risk of irAE is significantly more with dual-agent therapy (ipilimumab/nivolumab combination) and high disease burden. The only hematological parameter that was substantially different between the groups was the mean PLR, which was lower in the irAE group (200 vs. 257, $\mathrm{P}=0.04)$. None of the thresholds for baseline hematological factors, such as WBC $(10 \mathrm{k}), \operatorname{ALC}(0.6 \mathrm{k}, 1 \mathrm{k}$ or $2 \mathrm{k})$, and PLR (150), could predict the incidence of irAE. The number of doses of ICI people who had irAE was low when compared to non-irAE group. Other factors such as type of primary malignancy, lung cancer (more melanoma, $\mathrm{P}=0.06$ ); Stage of the diseases at the time of ICI therapy, stage IV disease (vs. stage II/III, $\mathrm{P}=0.06)$, and higher ALC $(\mathrm{P}=0.07)$ seem to have a considerable association with irAE even though the association is not statistically significant. More extensive studies may show conclusive results.

No significant difference was observed in the mean PLT/ 
Table 2. Comparison of Characteristics in irAE and Non-irAE Patients

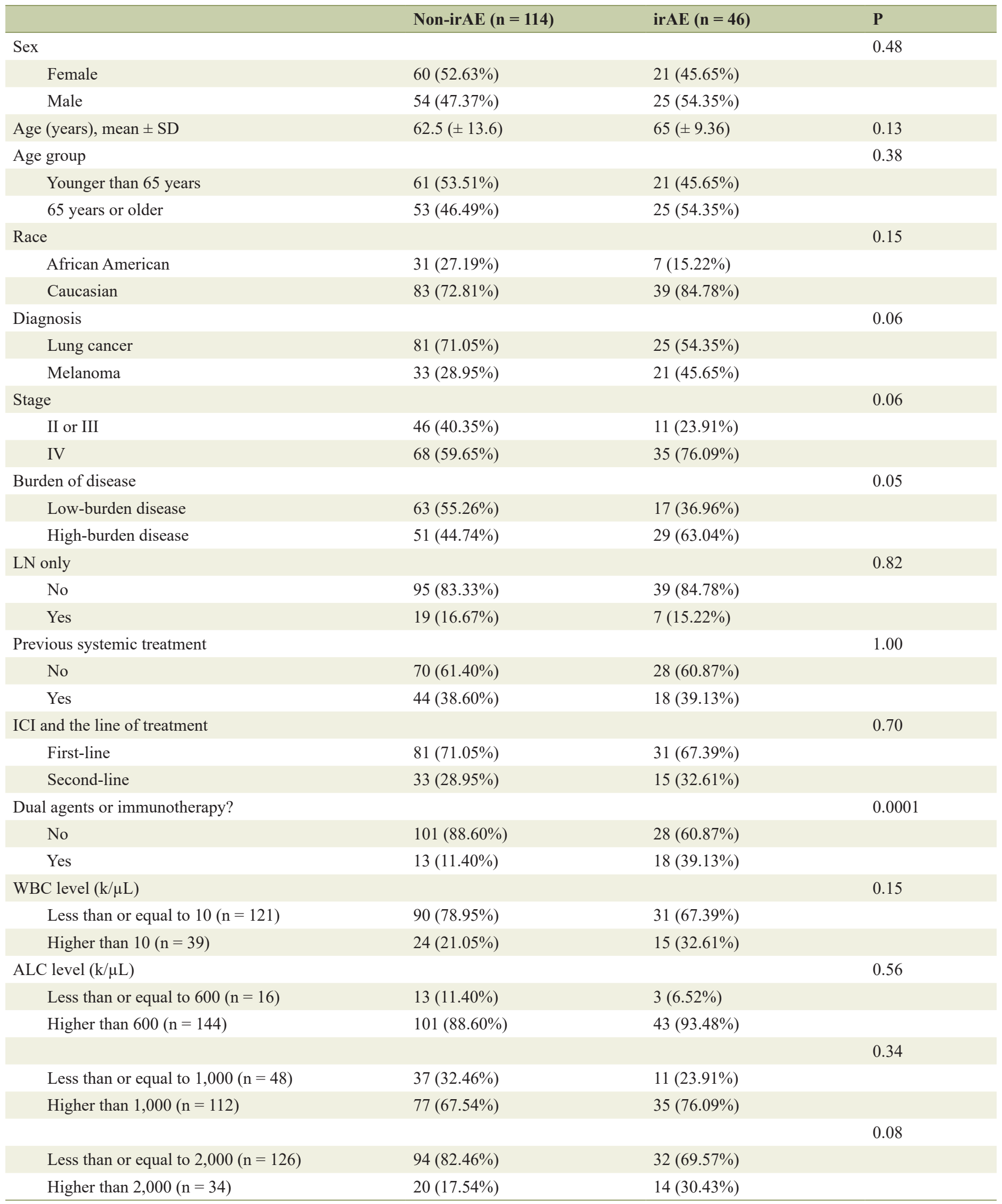


Table 2. Comparison of Characteristics in irAE and Non-irAE Patients - (continued)

\begin{tabular}{|c|c|c|c|}
\hline & Non-irAE $(n=114)$ & $\operatorname{irAE}(n=46)$ & $\mathbf{P}$ \\
\hline PLT/ALC ratio $(\mathrm{k} / \mu \mathrm{L})$ & & & 0.21 \\
\hline Less than or equal to $150(n=63)$ & $41(35.96 \%)$ & $22(47.83 \%)$ & \\
\hline $\mathrm{WBC}(\mathrm{k} / \mu \mathrm{L})$, mean $\pm \mathrm{SD}$ & $8.29 \pm 4.42$ & $8.34 \pm 2.67$ & 0.94 \\
\hline $\mathrm{Hb}(\mathrm{g} / \mathrm{dL})$, mean $\pm \mathrm{SD}$ & $11.89 \pm 2.14$ & $12.42 \pm 2.00$ & 0.14 \\
\hline $\operatorname{ALC}(\mathrm{k} / \mu \mathrm{L})$, mean $\pm \mathrm{SD}$ & $1.39 \pm 0.73$ & $1.78 \pm 1.36$ & 0.07 \\
\hline $\mathrm{ANC} / \mathrm{ALC}$, mean $\pm \mathrm{SD}$ & $5.34 \pm 5.19$ & $4.53 \pm 3.76$ & 0.27 \\
\hline $\mathrm{PLT} / \mathrm{ALC}$, mean $\pm \mathrm{SD}$ & $257.02 \pm 205.95$ & $200.62 \pm 139.11$ & 0.04 \\
\hline Number of cycles, mean \pm SD & $13.41 \pm 16.71$ & $6.91 \pm 7.67$ & 0.001 \\
\hline
\end{tabular}

SD: standard deviation; LN: Iymph node; ICl: immune checkpoint inhibitor; WBC: white blood cell; PLT: platelet; Hb: hemoglobin; ANC: absolute neutrophilic count; ALC: absolute lymphocyte count; irAE: immune-related adverse event.

ALC ratio by sex $(t$-test, $\mathrm{P}=0.6840)$, race $(t$-test, $\mathrm{P}=0.3801)$, and stage (ANOVA, $\mathrm{P}=0.7813$ ). No significant correlation was observed between the age at diagnosis and PLT/ALC ratio (Pearson's correlation coefficient $\mathrm{r}=-0.08, \mathrm{P}=0.3025$ ). Multivariate logistic regression was used to study association between the occurrence of adverse event and clinical and hematological factors (area under the ROC curve $=0.66$, correct classification rate $=73 \%)$. It showed $\mathrm{PLT} / \mathrm{ALC}$ ratio $(\mathrm{P}$ $=0.078)$ to be the most associated predictor in the presence of other clinical factors such as stage $(\mathrm{P}=0.1499)$, age $(\mathrm{P}=$ $0.2589)$, and sex $(\mathrm{P}=0.4116)$.

\section{Comparing baseline variables at the time of incidence of irAEs}

Although 46 patients had irAEs, blood work results at the irAE incidence were not available for one patient. Hence, the analysis of outcomes for 45 patients is reported here (Supplemental Material 3, www.jocmr.org). Among patients who had irAEs, the drop in ALC was significant (1.7 vs. $1.5, \mathrm{P}=0.03$ ) at the time of irAE when compared with the baseline ALC. The change in other hematological parameters (such as WBC, hemoglobin, ANC, platelet count, PLR, and NLR) was not significant. Post irAE, ICI was restarted in only 19 out of 46 ( $41 \%)$ of the irAE group, and five $(26 \%)$ of those patients had a recurrence of irAE.

\section{Discussion}

In the current clinical practice, there has been an exponential increase in the understanding of various types of immunotherapy, including ICIs, monoclonal antibodies, antibody-drug conjugates, and treatment vaccines in cancer treatment [26]. Given they have broad clinical indications and promising benefits, efforts must be made to make it more tolerable so that best outcomes can be achieved with fewer adverse events and hospitalizations [27].

This study cohort reflects the contemporary clinical practice with a mixture of patients treated with ICIs in the first and subsequent lines, combined with chemotherapy and alone. The influence of clinical factors, such as type of cancer, disease burden, line of ICI use, and combination therapy (with chemotherapy), on the PFS distribution in this cohort should be interpreted carefully as this is a heterogeneous group with: 1) different cancers (melanoma and lung cancer); 2) different stages; and 3) different histology (small cell vs. squamous cell vs. adenocarcinoma vs. melanoma). Prospective trials with such specific stratifications are needed to have meaningful conclusions. Moreover, this advantage in PFS did not result in a benefit for OS, and hence the use of this information for prediction purposes is doubtful.

Even in our cohort, demographic characteristics, such as age, sex, stage of the disease, or baseline blood counts, like ANC and ALC, or ratios, like PLR and NLR, did not influence OS and PFS. Pending large prospective trials proving otherwise, it is very hard to prove that baseline counts, such as ALC, NLR, or PLR, will impact the efficacy of ICI; and we may have to modify the treatment in terms of doses and the kind of therapy to be provided (cytotoxic T lymphocyte antigen 4 (CTLA-4) vs. PD-L1). Moreover, the WBCs of specific interest (e.g., lymphocyte count) can be affected by factors, such as infections, allergies, heart failure, nutritional deficiencies, and autoimmune diseases, which make them relatively unreliable markers to predict the response in ICI [28-31].

The common irAEs encountered with ICI that need hospital admission are dermatologic, gastrointestinal, hepatic, and endocrine [32]. In contrast to targeted therapy like epidermal growth factor receptor (EGFR)-inhibitors or anaplastic lymphoma kinase (ALK)-inhibitors, the actions of ICIs are non-specific as the activated cytotoxic T cells can attack nonmalignant tissue. This response may be altered based on the organ of origin and also disease burden. This can explain the considerable correlation (not statistically significant) of the in- 
creased risk of irAE in lung cancers (compared to melanoma) and metastatic cancers (in lung cancers and melanoma). On the other hand, patients with more than two metastatic organ sites are at significantly higher risk for irAE. The role of the primary tumor site and its related microenvironment was studied extensively for efficacy and survival, but very little literature is available on its influence on the incidence of irAE. The heterogeneity of this cohort allowed understanding it. This can potentially be translated to more cautionary use of ICIs in this subset of the population. It is not a surprise to see a higher incidence of irAE with dual-agent immunotherapy (as opposed to a single agent). The patients that tolerate more than seven to eight cycles seem to have fewer severe irAEs.

The general concept of immunotherapy is to induce an inflammatory reaction against foreign antigens. There is a lot of interest in studying the hematological parameters such as the WBCs or their differentials, like ALC or ANC and platelets. As individual counts can be affected by innumerable factors, ratios like PLR and NLR are also used to assess the baseline bone marrow status and the response to ICI. Among all the baseline hematological factors, such as WBC count, ALC, ANC, NLR, and platelet count, which were studied to predict the incidence of irAE, only the mean PLR was found to be significantly lower (200 vs. 250) in the group that had irAE. As PLR is a marker for inflammation, it may mean that in patients with irAE, the inflammation (and hence the immune response) is not adequate to mount a response and erratic enough to attack healthy cells and cause irAE. It is important to note that there is no baseline threshold of WBC, PLR, and ALC to predict the incidence of irAE confidently.

After initiating ICI, there are no reliable parameters that we can follow to predict the incidence of irAE (Supplement Material 3, www.jocmr.org). The drop in ALC from initiation of treatment to the occurrence of irAE is statistically significant, but the change is too small to follow and monitor. There seems to be a connection between bone marrow, response to ICI, and incidence of irAE. More extensive studies can help us in understanding this dynamic.

The management of irAEs in this cohort was according to the standard of care (as in NCCN guidelines) with methylprednisolone $1 \mathrm{mg} / \mathrm{kg}$ and was modified based on the patient's response with a scheduled tapering over 4 weeks or beyond. Re-initiation of the ICI in patients who recovered is usually at the physician's discretion. In our center, it is considered an appropriate decision to restart ICI in patients after complete resolution of symptoms and tapering doses of oral prednisone to at least $10 \mathrm{mg} /$ day. Post irAE, re-initiation of ICI (after recovery) is possible in a selected group of patients. Changing the class of ICI (CTLA-4 to PD-1/PD-L1, for example) was studied to see if that would reduce the chance of recurrence of irAE. Using a single agent (PD-L1 or PD-1) after the dualagent therapy (CTLA-4 + PD-1), to some extent, helped in preventing recurrences of irAE [33]. On the other hand, the use of CTLA-4 agents after the PD-1/PD-L1 agent increased the recurrences [34]. This study had too few patients (19) in whom ICI was re-initiated to draw any meaningful conclusions.

The retrospective model of the study restricted us from validating most of the results we discussed. Tumor biology was not the central focus of this study, which may have confounded most of the results, but many of the available studies on this topic are similar. We did not differentiate the type of ICI agent used other than dual agents or ipilimumab, but this is close to real-world data with growing applications of PD-1/ PD-L1 agents. Incorporating PD-L1 level of expression or tumor mutational burden (TMB) into the study might provide more information for future studies. In this study, grade $1 / 2$ and grade 3 irAE that did not require hospital admission were not studied. Including other cancers like colon cancer, renal cell carcinoma, head/neck cancers, hepatocellular, and gastric/esophageal cancers, in which the use of ICI is significant, would have made the results more convincing.

\section{Conclusions}

There is an unmet need for prospective trials incorporating innovative strategies to select appropriate candidates for ICI with a lower risk of irAE, and examine and validate the use of hematological parameters to predict the response and irAEs in solid tumors.

\section{Supplementary Material}

Suppl 1. PFS and OS (months) by patient characteristics.

Suppl 2. Distribution of progression-free survival (PFS).

Suppl 3. Comparison of blood count before starting IT and at the incidence of irAE.

\section{Acknowledgments}

None to declare.

\section{Financial Disclosure}

There was no specific funding source to be mentioned.

\section{Conflict of Interest}

The authors declare that they have no conflict of interest.

\section{Informed Consent}

All subjects provided written informed consents.

\section{Author Contributions}

$\mathrm{AM}$ and $\mathrm{OA}$ designed the study. AM drafted the manuscript and did critical editing. DE, AA, PP, and GS assisted and supported in data collection. MM assisted in the analysis with 
statistics. MK, JHH, RG assisted in designing the study. OA carefully supervised this manuscript preparation and writing.

\section{Data Availability}

Any inquiries regarding supporting data availability of this study should be directed to the corresponding author.

\section{Abbreviations}

irAEs: immune-related adverse events; ICIs: immune checkpoint inhibitors; PD-L1: programmed death-ligand 1; IL-17: interleukin 17; ICD: International Statistical Classification of Diseases; WBC: white blood cell; ALC: absolute lymphocyte count; NLR: neutrophil-to-lymphocyte ratio; PLR: platelet-tolymphocyte ratio

\section{References}

1. Hopkins AM, Kichenadasse G, McKinnon RA, Rowland A, Sorich MJ. Baseline tumor size and survival outcomes in lung cancer patients treated with immune checkpoint inhibitors. Semin Oncol. 2019;46(4-5):380-384.

2. Zhang L, Sun L, Yu J, Shan F, Zhang K, Pang X, Ma C, et al. Comparison of immune checkpoint inhibitors between older and younger patients with advanced or metastatic lung cancer: a systematic review and meta-analysis. Biomed Res Int. 2019;2019:9853701.

3. Rizvi NA, Hellmann MD, Snyder A, Kvistborg P, Makarov V, Havel JJ, Lee W, et al. Cancer immunology. Mutational landscape determines sensitivity to PD-1 blockade in nonsmall cell lung cancer. Science. 2015;348(6230):124-128.

4. Sahin IH, Akce M, Alese O, Shaib W, Lesinski GB, ElRayes B, Wu C. Immune checkpoint inhibitors for the treatment of MSI-H/MMR-D colorectal cancer and a perspective on resistance mechanisms. Br J Cancer. 2019;121(10):809-818.

5. Cottrell TR, Taube JM. PD-L1 and emerging biomarkers in immune checkpoint blockade therapy. Cancer J. 2018;24(1):41-46.

6. Eggermont AMM, Kicinski M, Blank CU, Mandala M, Long GV, Atkinson V, Dalle S, et al. Association between immune-related adverse events and recurrence-free survival among patients with stage III melanoma randomized to receive pembrolizumab or placebo: a secondary analysis of a randomized clinical trial. JAMA Oncol. 2020;6(4):519527.

7. Maher VE, Fernandes LL, Weinstock C, Tang S, Agarwal S, Brave M, Ning YM, et al. Analysis of the association between adverse events and outcome in patients receiving a programmed death protein 1 or programmed death ligand 1 antibody. J Clin Oncol. 2019;37(30):2730-2737.

8. Horvat TZ, Adel NG, Dang TO, Momtaz P, Postow MA, Callahan MK, Carvajal RD, et al. Immune-related adverse events, need for systemic immunosuppression, and effects on survival and time to treatment failure in patients with melanoma treated with ipilimumab at memorial sloan kettering cancer center. J Clin Oncol. 2015;33(28):31933198.

9. Shahabi V, Berman D, Chasalow SD, Wang L, Tsuchihashi $\mathrm{Z}, \mathrm{Hu} \mathrm{B}$, Panting L, et al. Gene expression profiling of whole blood in ipilimumab-treated patients for identification of potential biomarkers of immune-related gastrointestinal adverse events. J Transl Med. 2013;11:75.

10. Nakamura Y, Tanaka R, Maruyama H, Ishitsuka Y, Okiyama N, Watanabe R, Fujimoto $M$, et al. Correlation between blood cell count and outcome of melanoma patients treated with anti-PD-1 antibodies. Jpn J Clin Oncol. 2019;49(5):431-437.

11. Tarhini AA, Zahoor H, Lin Y, Malhotra U, Sander C, Butterfield LH, Kirkwood JM. Baseline circulating IL-17 predicts toxicity while TGF-beta1 and IL-10 are prognostic of relapse in ipilimumab neoadjuvant therapy of melanoma. J Immunother Cancer. 2015;3:39.

12. Dubin K, Callahan MK, Ren B, Khanin R, Viale A, Ling L, No D, et al. Intestinal microbiome analyses identify melanoma patients at risk for checkpoint-blockade-induced colitis. Nat Commun. 2016;7:10391.

13. Chaput N, Lepage P, Coutzac C, Soularue E, Le Roux $\mathrm{K}$, Monot C, Boselli L, et al. Baseline gut microbiota predicts clinical response and colitis in metastatic melanoma patients treated with ipilimumab. Ann Oncol. 2017;28(6):1368-1379.

14. Kimbara S, Fujiwara Y, Iwama S, Ohashi K, Kuchiba A, Arima H, Yamazaki N, et al. Association of antithyroglobulin antibodies with the development of thyroid dysfunction induced by nivolumab. Cancer Sci. 2018;109(11):35833590.

15. Sullivan RJ, Hamid O, Gonzalez R, Infante JR, Patel MR, Hodi FS, Lewis KD, et al. Atezolizumab plus cobimetinib and vemurafenib in BRAF-mutated melanoma patients. Nat Med. 2019;25(6):929-935.

16. Minor DR, Puzanov I, Callahan MK, Hug BA, Hoos A. Severe gastrointestinal toxicity with administration of trametinib in combination with dabrafenib and ipilimumab. Pigment Cell Melanoma Res. 2015;28(5):611-612.

17. Brahmer JR, Lacchetti C, Schneider BJ, Atkins MB, Brassil KJ, Caterino JM, Chau I, et al. Management of immune-related adverse events in patients treated with immune checkpoint inhibitor therapy: American Society of Clinical Oncology Clinical Practice Guideline. J Clin Oncol. 2018;36(17):1714-1768.

18. Simonaggio A, Michot JM, Voisin AL, Le Pavec J, Collins M, Lallart A, Cengizalp G, et al. Evaluation of Readministration of Immune Checkpoint Inhibitors After Immune-Related Adverse Events in Patients With Cancer. JAMA Oncol. 2019;5(9):1310-1317.

19. Menzies AM, Johnson DB, Ramanujam S, Atkinson VG, Wong ANM, Park JJ, McQuade JL, et al. Anti-PD-1 therapy in patients with advanced melanoma and preexisting autoimmune disorders or major toxicity with ipilimumab. Ann Oncol. 2017;28(2):368-376.

20. Weber JS, Kudchadkar RR, Yu B, Gallenstein D, Horak CE, Inzunza HD, Zhao X, et al. Safety, efficacy, and bio- 
markers of nivolumab with vaccine in ipilimumab-refractory or -naive melanoma. J Clin Oncol. 2013;31(34):43114318.

21. Santini FC, Rizvi H, Plodkowski AJ, Ni A, Lacouture ME, Gambarin-Gelwan M, Wilkins O, et al. Safety and Efficacy of Re-treating with Immunotherapy after Immune-Related Adverse Events in Patients with NSCLC. Cancer Immunol Res. 2018;6(9):1093-1099.

22. Arbour KC, Mezquita L, Long N, Rizvi H, Auclin E, Ni A, Martinez-Bernal G, et al. Impact of baseline steroids on efficacy of programmed cell death-1 and programmed death-ligand 1 blockade in patients with non-small-cell lung cancer. J Clin Oncol. 2018;36(28):2872-2878.

23. Common Terminology Criteria for Adverse Events (CTCAE) - CTCAE_v5_Quick_Reference_5x7.pdf. 2020. Available from: https://ctep.cancer.gov/protocolDevelopment/electronic_applications/docs/CTCAE_v5_Quick_ Reference $5 \times 7 . \overline{p d f}$.

24. Diehl A, Yarchoan M, Hopkins A, Jaffee E, Grossman SA. Relationships between lymphocyte counts and treatment-related toxicities and clinical responses in patients with solid tumors treated with PD-1 checkpoint inhibitors. Oncotarget. 2017;8(69):114268-114280.

25. Ho WJ, Yarchoan M, Hopkins A, Mehra R, Grossman S, Kang H. Association between pretreatment lymphocyte count and response to PD1 inhibitors in head and neck squamous cell carcinomas. J Immunother Cancer. 2018;6(1):84.

26. Yang Y. Cancer immunotherapy: harnessing the immune system to battle cancer. J Clin Invest. 2015;125(9):3335-
3337.

27. Alkharabsheh O, Kannarkatt P, Kannarkatt J, Karapetyan L, Laird-Fick HS, Al-Janadi A. An overview of the toxicities of checkpoint inhibitors in older patients with cancer. J Geriatr Oncol. 2018;9(5):451-458.

28. Leandro-Merhi VA, Braz VN, Aquino JL. Is total lymphocyte count related to nutritional markers in hospitalized older adults? Arq Gastroenterol. 2017;54(1):79-82.

29. George TI. Malignant or benign leukocytosis. Hematology Am Soc Hematol Educ Program. 2012;2012:475-484.

30. Lu Z, Li J, Ji J, Gu Z, Da Z. Altered peripheral lymphocyte subsets in untreated systemic lupus erythematosus patients with infections. Braz J Med Biol Res. 2019;52(4):e8131.

31. Charach G, Grosskopf I, Roth A, Afek A, Wexler D, Sheps D, Weintraub M, et al. Usefulness of total lymphocyte count as predictor of outcome in patients with chronic heart failure. Am J Cardiol. 2011;107(9):1353-1356.

32. Wang DY, Salem JE, Cohen JV, Chandra S, Menzer C, Ye F, Zhao S, et al. Fatal toxic effects associated with immune checkpoint inhibitors: a systematic review and meta-analysis. JAMA Oncol. 2018;4(12):1721-1728.

33. Abu-Sbeih H, Ali FS, Naqash AR, Owen DH, Patel S, Otterson GA, Kendra K, et al. Resumption of immune checkpoint inhibitor therapy after immune-mediated colitis. J Clin Oncol. 2019;37(30):2738-2745.

34. Pollack MH, Betof A, Dearden H, Rapazzo K, Valentine I, Brohl AS, Ancell KK, et al. Safety of resuming anti-PD-1 in patients with immune-related adverse events (irAEs) during combined anti-CTLA-4 and anti-PD1 in 